\title{
Genomic characterization of asymptomatic Escherichia coli isolated from the neobladder
}

\begin{abstract}
Correspondence
David A. Rasko

drasko@som.umaryland.edu
\end{abstract}

Received 26 June 2010

Revised 20 December 2010

Accepted 14 January 2011
Jason W. Sahl, ${ }^{1} \dagger$ Amanda L. Lloyd, ${ }^{2} \dagger$ Julia C. Redman, ${ }^{1}$

Thomas A. Cebula, ${ }^{3}$ David P. Wood, ${ }^{4}$ Harry L. T. Mobley ${ }^{2}$

and David A. Rasko ${ }^{1,5}$

${ }^{1}$ Institute for Genome Sciences, University of Maryland School of Medicine, Baltimore, MD 21201, USA

${ }^{2}$ Department of Microbiology and Immunology, University of Michigan Medical School, 1150 West Medical Center Drive, 5641 Medical Science II, Ann Arbor, MI 48109, USA

${ }^{3}$ Johns Hopkins University, Department of Biology, 3400 North Charles Street, Baltimore, MD 21218, USA

${ }^{4}$ University of Michigan Medical School, Department of Urology, 1500 East Medical Center Drive, Ann Arbor, Ml 48109, USA

${ }^{5}$ Department of Microbiology and Immunology, University of Maryland School of Medicine, Baltimore, MD 21201, USA

The replacement of the bladder with a neobladder made from ileal tissue is the prescribed treatment in some cases of bladder cancer or trauma. Studies have demonstrated that individuals with an ileal neobladder have recurrent colonization by Escherichia coli and other species that are commonly associated with urinary tract infections; however, pyelonephritis and complicated symptomatic infections with ileal neobladders are relatively rare. This study examines the genomic content of two $E$. coli isolates from individuals with neobladders using comparative genomic hybridization $(\mathrm{CGH})$ with a pan-E. coli/Shigella microarray. Comparisons of the neobladder genome hybridization patterns with reference genomes demonstrate that the neobladder isolates are more similar to the commensal, laboratory-adapted $E$. coli and a subset of enteroaggregative $E$. coli than they are to uropathogenic $E$. coli isolates. Genes identified by CGH as exclusively present in the neobladder isolates among the 30 examined isolates were primarily from large enteric isolate plasmids. Isolations identified a large plasmid in each isolate, and sequencing confirmed similarity to previously identified plasmids of enteric species. Screening, via PCR, of more than 100 isolates of $E$. coli from environmental, diarrhoeagenic and urinary tract sources did not identify neobladder-specific genes that were widely distributed in these populations. These results taken together demonstrate that the neobladder isolates, while distinct, are genomically more similar to gastrointestinal or commensal $E$. coli, suggesting why they can colonize the transplanted intestinal tissue but rarely progress to acute pyelonephritis or more severe disease.
†These authors contributed equally to this work.

Abbreviations: $\mathrm{CGH}$, comparative genomic hybridization; EAEC, enteroaggregative E. coli; EHEC, enterohaemorrhagic E. coli; EIEC, enteroinvasive E. coli; EPEC, enteropathogenic E. coli; ETEC, enterotoxigenic E. coli; gDNA, genomic DNA; MEV, Multiple Experiment Viewer; MLST, multi-locus sequence typing; SPATE, serine protease autotransporter of Enterobacteriaceae; UPEC, uropathogenic E. coli; UTI, urinary tract infection.

Comparative genomic hybridization data can be located at www.ncbi.nlm. nih.gov/geo/ under series number GSE27326.

A supplementary figure and five supplementary tables are available with the online version of this paper.

\section{INTRODUCTION}

The surgical reconstruction of the lower urinary tract is required in cases of bladder cancer, neurological disorder of the bladder (neuropathic bladder), trauma or other clinical indications for the removal of bladder (cystectomy) (Davidsson et al., 2000; Falagas \& Vergidis, 2005; Wullt et al., 2004). The bladder can be replaced via a number of surgical techniques and with a variety of tissues, the most common being the construction of an orthotopic neobladder with ileal or colonic tissue (Falagas \& Vergidis, 2005). In the construction of a neobladder, a section of the ileal or colonic tissue is excised and formed into a receptacle for the draining ureters (Falagas \& Vergidis, 
2005). The connection of the intestinal tissue with the ureter is a key feature of the surgery to prevent reflux of the urine into the kidney, potentially leading to pyelonephritis, kidney scarring and sepsis (Kristjánsson et al., 1995a, b).

Bacteriuria is common among individuals who have had neobladder surgery, with Escherichia coli, Proteus spp., Klebsiella spp. and Enterobacter faecalis being the most common bacterial species present (Iwakiri et al., 2002; Wullt et al., 2004). The physical processes associated with the construction of a neobladder may add confounding factors to the attribution of the origin of the bacteriuria; compared with the bladder, the neobladder has altered innervation, which subsequently alters the voiding pattern and bladder retention volume (Iwakiri \& Freiha, 1993; Iwakiri et al., 2002; Wullt et al., 2004). Additionally, any innate immunity that was associated with bladder epithelium and associated lower urinary tract is no longer present, although some innate immune attributes of the ileal tissue may contribute to protection (Iwakiri et al., 1993, 2002; Wullt et al., 2004). Studies have postulated that the reason for the lack of widespread upper urinary tract involvement is that the neobladder is a low-pressure environment and thus there is not sufficient pressure to cause vesicouretal reflux of the bacteria into the upper urinary tract (Shaaban et al., 1992a, b).

Long-term studies have demonstrated that many patients with neobladders are colonized continually with the aforementioned bacterial species commonly associated with urinary tract infection (Wullt et al., 2004); however, the rates of symptomatic infection are relatively low, as measured by pyelonephritis and systemic infection associated with colonization (Kristjánsson et al., 1995a, b). The reasons for the lack of symptomatic presentation are not well understood. Åkerlund et al. (1994) suggested that aggressive treatment with antibiotics is not required and the observed bacteriuria should be considered part of colonization by normal microbiota (Åkerlund et al., 1994). Conflicting evidence related to the pathogenic potential of E. coli colonizing the urinary tract has been presented in the literature. Studies by Schlager et al. $(2000,2008)$ demonstrate that the E. coli isolates from neobladder and neurogenic cases are more similar to isolates from individuals with uncomplicated or asymptomatic urinary tract infection using multi-locus sequence typing (MLST) and directed PCR. Conversely, Keegan et al. (2000, 2003) used pulsed-field gel electrophoresis and focused examination of uropathogenic virulence factors to suggest that neobladder isolates have a greater pathogenic potential than faecal isolates. In contrast to these previous studies, the current study takes a complete genomic approach to the examination of $E$. coli isolates from neobladder patients.

The E. coli and Shigella species represent one of the most studied organisms on a genomic scale. As of December 2010, more than 150 genome projects have been completed, yielding either a finished genome sequence (closed to a high standard) or a genome sequence in draft form (multiple high quality contigs) (see http://genomesonline. org/ and http://www.ncbi.nlm.nih.gov/sites/genome). With new genomic technologies becoming more widespread, there are multiple projects that will soon add hundreds of draft genomes from this taxonomic group. Pan-genome studies from publicly available genomes have identified approximately 2200 genes that are common to all E. coli; furthermore, the pan-genome of this organism is open, suggesting that there is a constant influx of genetic material into the species (Rasko et al., 2008; Touchon et al., 2009). Molecular models (Tettelin et al., 2008) of the conserved and unique genes in each of the genomes have suggested that $\sim 300$ new genes could be identified in each new sequenced genome (Rasko et al., 2008).

Comparative genomic hybridization has been used previously to determine genetic composition in closely related isolates of E. coli and Shigella (Fukiya et al., 2004; Nie et al., 2006). In the current study, a pan-genome microarray for E. coli and Shigella containing the genomes of 32 diverse E. coli and Shigella as well as 46 related plasmid sequences (Fang et al., 2010) was utilized. This pan-genome microarray design is a significant advance over previous array designs, as it contains multiple genomes and allows for the examination of horizontal gene flow between the pathogenic variants (pathovars) of E. coli and Shigella species included on the array.

The primary goal of this study was to determine whether the environment (urine-filled bladder) or the tissue (gastrointestinal origin) is the defining feature in the colonization of the neobladder by E. coli. Overall, these genomic studies demonstrate that isolates from neobladder sources are more similar to commensal and laboratory-adapted isolates, and to one heterogeneous pathovar of diarrhoeagenic E. coli, than they are to isolates from uropathogenic sources. This suggests that tissue tropism for the gastrointestinal tract overrides the selective forces of the urine in the colonization processes of the neobladder by E. coli.

\section{METHODS}

Strain isolation. Isolates were obtained from the urine of asymptomatic individuals who had undergone bladder removal (cystectomy) due to cancer and then received neobladder reconstruction utilizing ileal tissue. Clinical parameters for each of these subjects are included in Table 1. Samples were collected in accordance with the IRB protocol number HUM00004950 at the University of Michigan Medical School. Bacterial isolates were selected by growth on selective media and presumptively identified as E. coli. The identity of the isolates as $E$. coli were confirmed by amplification and sequencing of the $16 \mathrm{~S}$ rRNA gene by conserved universal primers (Lane et al., 1985). The isolates were also sent to the Pennsylvania State University E. coli Reference Center for the determination of $\mathrm{O}$ - and $\mathrm{H}$-antigens as well as toxin and adhesin typing (http://ecoli. cas.psu.edu/, Table 1).

Genomic DNA isolation for hybridization. Efforts were made to minimize the number of passages of all isolates. Bacterial cultures were grown overnight from a population in $50 \mathrm{ml}$ Luria broth (LB) and genomic DNA was isolated according to standard methods (Ge \& 
Table 1. Clinical and microbiological features associated with neobladder isolates

\begin{tabular}{|lcccccccc|}
\hline Sample & Symptom status & $\begin{array}{c}\text { Sex of } \\
\text { patient }\end{array}$ & $\begin{array}{c}\text { Age of } \\
\text { patient }\end{array}$ & $\begin{array}{c}\text { Time since last } \\
\text { symptomatic UTI }\end{array}$ & $\begin{array}{c}\text { Time since } \\
\text { neobladder }\end{array}$ & $\begin{array}{c}\text { Voiding } \\
\text { status }\end{array}$ & $\begin{array}{c}\text { Sero } \\
\text { group }\end{array}$ & $\begin{array}{c}\text { H-antigen } \\
\text { NB001 }\end{array}$ \\
Asymptomatic & Male & 70 & Not in post-surgical time & $<1$ year* & Self & NT $\dagger$ \\
NB002 & Asymptomatic & Male & 69 & 6 months $\ddagger$ & $\sim 10$ years & Self & NT & 9 \\
\hline
\end{tabular}

${ }^{\star}$ Date of neobladder January 2008; date of sample 29 September 2008.

$\dagger \mathrm{NT}$, non-typable.

$\ddagger$ Last UTI April 2008.

Taylor, 1992). Briefly, bacterial cells were concentrated by centrifugation, washed and suspended in isolation buffer $(0.15 \mathrm{M}$ Tris, $0.1 \mathrm{M}$ EDTA, pH 8.0). SDS was added to $1 \%(\mathrm{v} / \mathrm{v})$ final concentration and allowed to incubate for $1 \mathrm{~h}$ at $55{ }^{\circ} \mathrm{C}$ or until the solution cleared. Two volumes of phenol/chloroform/isoamyl alcohol (25:24:1) were added and briefly mixed by vortexing. The resulting solution was separated by centrifugation at $12000 \mathrm{~g}$ for $15 \mathrm{~min}$ at $4{ }^{\circ} \mathrm{C}$. The aqueous layer (top) was removed to a new tube and mixed with 2 volumes of chloroform. The mixture was separated by centrifugation at $12000 \mathrm{~g}$ for $15 \mathrm{~min}$ at $4{ }^{\circ} \mathrm{C}$ and the aqueous layer (top) was transferred to a clean tube. The aqueous layer was then extracted with at least 10 volumes of ice-cold ethanol. The precipitated DNA was spooled out of the mixture and suspended in TE $(10 \mathrm{mM} \mathrm{Tris} / \mathrm{HCl}$, $1 \mathrm{mM}$ EDTA, $\mathrm{pH}$ 8.0). The purified mixture was further digested with RNase overnight at $37{ }^{\circ} \mathrm{C}$ and reprecipitated the following day with 0.1 volumes of $3 \mathrm{M}$ sodium acetate and 10 volumes of ice-cold ethanol. The pellet was allowed to air dry and then dissolved in a minimal volume of nuclease-free water (Ambion). The quantity and quality of genomic DNA (gDNA) was verified by gel electrophoresis, nanodrop and picogreen assay.

Comparative genome hybridization (CGH). gDNA was prepared for CGH as previously described (http://www.affymetrix.com/ support) with the following modifications. Approximately $7.5 \mu \mathrm{g}$ purified gDNA isolated as described above was digested with the DNAfree DNase kit (ABI) using timed samples to provide a sample with a size range of between 20 and $200 \mathrm{bp}$. DNase was inactivated with the Inactivation Reagent provided with the kit. Sample ranges were determined by gel electrophoresis on a 4-20\% gradient gel (BioRad) with Low MW Ladder (Invitrogen). Digested DNA was labelled with biotin by the addition of biotin-11-ddATP (Perkin-Elmer), $2 \mu \mathrm{l}$ terminal transferase enzyme (Promega) and appropriate buffers. The digests were incubated for $2 \mathrm{~h}$ at $37{ }^{\circ} \mathrm{C}$ and the labelling was quenched with $0.5 \mathrm{M}$ EDTA. Verification of label incorporation was accomplished by mixing the biotin-labelled DNA with Immunopure NeutrAvidin (Pierce), incubation at room temperature for $5 \mathrm{~min}$, electrophoresis on a $4-20 \%$ gradient gel (Bio-Rad) and visualization. A clear shift in size distribution from unlabelled to labelled DNA was verified prior to hybridization.

Hybridization of the digested probe to the pan-genome array. The pan-genome microarrays used in this study were designed by FDA-Laurel and contain the genomes of 32 diverse E. coli and Shigella spp. as well as 46 related plasmid sequences (a description and distribution of the microarray are available via the website at the Pathogen Functional Genomics Resources Center, http://pfgrc.jcvi. org/index.php/home/full_news/current/2009_1_9.html; Fang et al., 2010). The microarrays were stored and processed according to the manufacturer's suggestions. Briefly, microarrays were allowed to warm to room temperature for $30 \mathrm{~min}$. The OligoB2 mixture (Affymetrix) was heated to $65{ }^{\circ} \mathrm{C}$ for $5 \mathrm{~min}$ then mixed with the labelled and verified gDNA, DMSO and hybridization buffer included in the Affymetrix Hybridization, Wash and Stain kit. This solution was further denatured at $94{ }^{\circ} \mathrm{C}$ for $5 \mathrm{~min}$. During this incubation the microarrays were equilibrated with prehybridization buffer and placed in the Affymetrix hybridization oven at $45^{\circ} \mathrm{C}$ for $10 \mathrm{~min}$, rotating at 60 r.p.m. The labelled DNA was then hybridized to the microarray for $16 \mathrm{~h}$ at $45{ }^{\circ} \mathrm{C}$, rotating at 60 r.p.m.

Microarray washing and staining. Microarrays were washed and stained according to manufacturer's specifications using two stain cycles and the prokaryotic washing protocol (http://www.affymetrix. com).

Data analysis. Initial data analysis was performed with the Gene Chip Operating System (GCOS) suite of tools provided by Affymetrix. Additional analysis utilized the Affymetrix power tools (APT) software (http://www.affymetrix.com/partners_programs/programs/developer/ tools/powertools.affx). The data analysis parameters have been established empirically using known reference genomes included on the array. Within the APT, the MAS5 algorithm (Hubbell et al., 2002; Liu et al., 2002) was utilized with the perfect match and mismatch calculations and a Tau of 0.150 to detect which probes were present or absent (command $=$ apt-probeset-summarize $-\mathrm{a}$ pm-mm,mas5-detect. calls $=1$. pairs $=1$. Tau $=0.150$-d FDA_ECSGa520423F.cdf -0 mas5_ Analysis.dir --cel-files cel_file_list.txt). Features that were present or absent in all samples were removed from further analysis. The resulting features, known as the variable gene set, were analysed using Multiple Experiment Viewer v4.5 (MEV; http://www.tm4.org/mev/). Custom Perl scripts written by the authors were used to complete further genomic analyses.

Plasmid preparation. Each of the neobladder isolates was grown in $150 \mathrm{ml} \mathrm{LB}$ overnight and subjected to plasmid isolation using the Phase Prep BAC DNA kit (Sigma NA0100-1KT). Each of the plasmid preparations was submitted for sequencing at the Institute for Genome Sciences Genomics Resource Center. Each sample was part of a multiplexed lane of sequencing on the Solexa/Illumina platform. A total of $14800050 \mathrm{bp}$ paired end sequences were obtained from plasmid sequences from both neobladder plasmid preparations. De novo assembly was performed using Velvet (Zerbino \& Birney, 2008) with parameters optimized by the VelvetOptimizer http://www. bioinformatics.net.au/software.velvetoptimiser.shtml. The resulting contigs and scaffolds were compared by BLASTX (Altschul et al., 1990) to the nr database at NCBI, containing all nucleotide data in GenBank, RefSeq Nucleotides, EMBL (European nucleotide database), DDBJ (Japanese nucleotide database) and PDB (Protein Data Bank) sequences, but not expressed sequence tags (EST), Sequence Tagged Sites (STS), Genome Survey Sequences (GSS), or incomplete high-throughput genome sequencing projects, to verify homology to previously annotated plasmid sequence. Contigs were mapped to large $(>50 \mathrm{~kb})$ plasmids from E. coli, Shigella and Salmonella using MUMmer (Delcher et al., 2002, 2003; Kurtz et al., 2004). Plasmid sequences from the two isolates were aligned to one another 
with Mugsy (Angiuoli \& Salzberg, 2011) to identify regions of conservation.

E. coli genomic isolation for PCR screening. Genomic DNA was obtained from 133 isolates belonging to three separate sample origins: 21 isolates from the diarrhoeagenic E. coli set (DECA), the ECOR set of 72 isolates from the STEC Center (http://www.shigatox.net/stec/ cgi-bin/index), which includes 11 isolates of uropathogenic E. coli, and an additional $40 \mathrm{E}$. coli isolates from urinary tract infection (UTI) sources (Mobley et al., 1990). Cultures were grown overnight in LB and genomic DNA was isolated using the QIAamp DNA Mini kit (Qiagen) and quantified using Nanodrop, and standard quantities (50 ng) were added to PCRs.

\begin{abstract}
Amplification and analysis of amplicon-based screening of diarrhoeagenic and uropathogenic $E$. coli. PCR primer sequences and targets are listed in Supplementary Table S1, available with the online version of this paper. Primers were designed with a melting point between $68{ }^{\circ} \mathrm{C}$ and $70{ }^{\circ} \mathrm{C}$ and to amplify products using a touchdown reaction strategy consisting of five cycles of $95{ }^{\circ} \mathrm{C}$ for $30 \mathrm{~s}, 70{ }^{\circ} \mathrm{C}$ for $1 \mathrm{~min}$, five cycles of $95{ }^{\circ} \mathrm{C}$ for $30 \mathrm{~s}, 68^{\circ} \mathrm{C}$ for $1 \mathrm{~min}$, and 30 cycles of $95{ }^{\circ} \mathrm{C}$ for $30 \mathrm{~s}, 66{ }^{\circ} \mathrm{C}$ for $1 \mathrm{~min}$ and $72{ }^{\circ} \mathrm{C}$ for $5 \mathrm{~min}$ using AmpliTaq Gold 360 (Applied Biosystems) reagents. Each of the amplified products was designed to a different size so that the reactions could be multiplexed into a single reaction per sample. Reference genomic and sample DNAs were assayed under recommended conditions with a $2 \mathrm{mM} \mathrm{MgCl}_{2}$ concentration. Each neobladder group (NB001-specific, NB002-specific, and NB001 and NB002) was multiplexed and assayed in a single tube, increasing magnesium concentration to $2.5 \mathrm{mM}$, dNTP to $0.3 \mu \mathrm{M}$ each and doubling Taq concentration. Amplicons were electrophoresed on $1.5 \%$ agarose gels with ethidium bromide and visualized on a BioRad GelDoc system.
\end{abstract}

\section{RESULTS}

\section{Clinical features of the subjects with neobladders}

Two bacterial isolates were cultured from the urine of individuals with neobladders. Both subjects were male, $>69$ years of age, had received neobladders due to cancer and could void urine from the neobladder independent of catheterization. Both were asymptomatic at the time of isolate collection and had not had a symptomatic urinary tract infection in the 6 months prior to obtaining the culture. Interestingly, the NB001 isolate was provided from a subject who had received a neobladder approximately 8 months earlier, whereas the NB002 isolate was from a subject who had had a neobladder for $\sim 10$ years. These isolates provide a baseline for the genomic analysis of $E$. coli isolates from the neobladder.

\section{Microbiological features of isolates from neobladder samples}

The bacterial isolates from the two cultures presumptively identified to be E. coli were confirmed using comparative analysis of $16 \mathrm{~S}$ rRNA gene sequences against public databases (data not shown). Serotyping of the $\mathrm{O}-$ and $\mathrm{H}-$ antigens demonstrated that each of these isolates was nontypable for the O-antigen, but NB001 and NB002 were $\mathrm{H}$ antigen 4 and 9 positive, respectively (Table 1). Additional molecular typing at the E. coli Reference Center at the Pennsylvania State University (http://ecoli.cas.psu.edu/) for common diarrhoeagenic E. coli toxins [including enterotoxigenic E. coli (ETEC) eltA (LT), estA (STa), estb (STb); enterohaemorrhagic E. coli (EHEC) Shiga toxin $s t x A, s t x b$ and cytotoxic necrotizing factors (cnfl, cnf2)] as well as enteropathogenic E. coli (EPEC) bundle-forming pilus $(b f p)$ and EPEC/EHEC intimin (eae) did not identify any of these classic virulence factors.

\section{Comparative genomic hybridization of reference and neobladder isolates}

Comparative genomic hybridization to the pan-genome $E$. coli and Shigella microarray was used to determine the genetic relatedness of the neobladder isolates to a panel of reference $E$. coli isolates (Table 2). This reference isolate set contains representatives of each of the diarrhoeagenic $E$. coli pathovars, a representative of each Shigella species and laboratory-adapted as well as human commensal E. coli isolates. Through analysis of all 23090 features on the

Table 2. Isolates hybridized to the pan-genome array

\begin{tabular}{|c|c|c|}
\hline Strain & Pathovar & Genomic reference \\
\hline EDL933 & EHEC & Perna et al. (2001) \\
\hline Sakai & EHEC & Hayashi et al. (2001) \\
\hline 8624 & EHEC & D. A. Rasko, unpublished \\
\hline OK1114 & EHEC & This work \\
\hline TX1999 & EHEC & This work \\
\hline B171 & EPEC & Rasko et al. (2008) \\
\hline E110019 & EPEC & Rasko et al. (2008) \\
\hline E22 & EPEC & Rasko et al. (2008) \\
\hline Ec042 & EAEC & Unpublished \\
\hline $101-1$ & EAEC & Rasko et al. (2008) \\
\hline 55989 & EAEC & Touchon et al. (2009) \\
\hline $17-2$ & EAEC & This work \\
\hline JM221 & EAEC & This work \\
\hline B7A & ETEC & Rasko et al. (2008) \\
\hline E24377A & ETEC & Rasko et al. (2008) \\
\hline HS & Commensal & Rasko et al. (2008) \\
\hline $\mathrm{K}-12$ & $\mathrm{Lab}$ & Blattner et al. (1997) \\
\hline CFT073 & UPEC & Welch et al. (2002) \\
\hline F11 & UPEC & Rasko et al. (2008) \\
\hline UTI536 & UPEC & Hochhut et al. (2006) \\
\hline F24 & UPEC & Mobley et al. (1990) \\
\hline F38 & UPEC & Mobley et al. (1990) \\
\hline CFT01 & UPEC & Mobley et al. (1990) \\
\hline NB001 & Neobladder & This work \\
\hline NB002 & Neobladder & This work \\
\hline EIEC_53638 & EIEC $^{*}$ & D. A. Rasko, unpublished \\
\hline SD1_1617 & Shigella dysenteriae & Hale et al. (1984) \\
\hline SF_2457T & Shigella flexneri & Wei et al. (2003) \\
\hline SS_53G & Shigella sonnei & Unpublished \\
\hline SB_BS512 & Shigella boydii & D. A. Rasko, unpublished \\
\hline
\end{tabular}

${ }^{\star}$ EIEC, enteroinvasive E. coli. 
microarray, 4471 features $(19.36 \%)$ were identified that were shared by all 30 isolates hybridized in this study and 1305 features $(5.65 \%)$ that were absent in all 30 isolates examined. The remaining 17314 features displayed a variable pattern of presence or absence. The variable gene set of the 30 isolates included in this study was clustered with an untrained hierarchical clustering method with Pearson correlation and 1000 iterations in MEV (http:// www.tm4.org/mev/). This analysis separated isolates into four distinct clusters that are similar to the pathovar evolutionary histories and phylogenetic groups as previously described (Donnenberg \& Whittam, 2001; Whittam, 1993; Whittam et al., 1983). In Fig. 1, the four clusters are uropathogenic E. coli (UPEC) (coloured in gold; upper right), Shigella and O157: H7 EHEC (coloured in burgundy and brown, respectively; upper left), nonO157 : H7 EHEC, EPEC and ETEC (coloured brown, blue and red respectively; lower right) and laboratory-adapted, commensal, neobladder isolates and three out of five enteroaggregative E. coli (EAEC) (coloured black, red and green; lower left). EAEC isolates are represented in three of the four clusters, but not in the cluster that contains the UPEC isolates. This is most probably due to the diverse nature of the EAEC pathovar as previously determined by multi-locus sequence typing and phenotypic methods (Czeczulin et al., 1999). Notably, both neobladder isolates examined are within the commensal/laboratory-adapted cluster (Fig. 1, arrows) and not within the UPEC cluster.
These data demonstrate that the neobladder isolates are more similar to commensal and/or diarrhoeagenic E. coli than they are to UPEC isolates on a genomic level.

\section{Identification of UPEC virulence factors in the CGH data}

On a whole-genome scale the neobladder isolates did not appear similar to UPEC (Fig. 1). To determine if the neobladder isolate diversity extended to UPEC virulence factors, a defined set of UPEC virulence factors previously identified (Lloyd et al., 2007, 2009) was examined. In total, 172 UPEC virulence factor gene features were identified, including serine protease autotransporters of Enterobacteriaceae (SPATEs), genes involved in the acquisition or sequestration of iron, adhesins and pathogenicity islands. Fig. 2 demonstrates that the UPEC-associated virulence factors are conserved in the UPEC isolates. Conversely, these UPEC virulence factors are not consistently identified in non-UPEC isolates. This finding could also be extended to the various pathovars represented in the hybridization data (see Supplementary Table S2). The difference between the UPEC virulence factor profile in each of the other pathovars was statistically significant, as determined by a Student's $t$-test $(P<0.05)$ with the exception of the neobladder isolates $(P=0.058)$. This borderline $P$-value is most probably due to the relatively low number of isolates in the neobladder group.

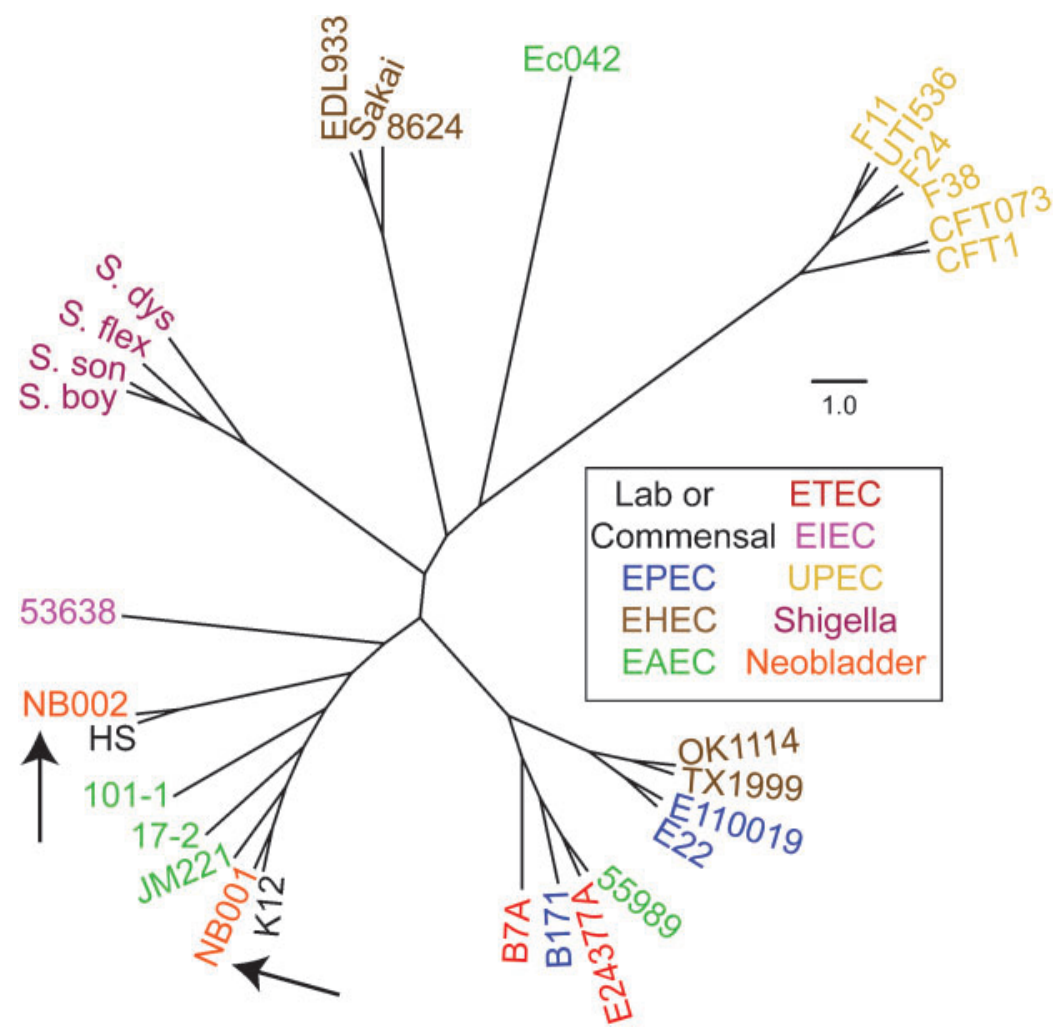

Fig. 1. Hierarchical cluster diagram displaying the relatedness of the reference and neobladder isolates. Using only the variable regions from the $\mathrm{CGH}$ studies the samples were analysed by sample with hierarchical clustering including support trees with Pearson correlation including 1000 iterations. Isolates of $E$. coli and Shigella are coloured according to pathovar. The arrows indicate the location of the neobladder isolates relative to the other isolates screened. The neobladder isolates cluster in a group that includes EAEC, commensal and laboratory isolates but no UPEC isolates. 


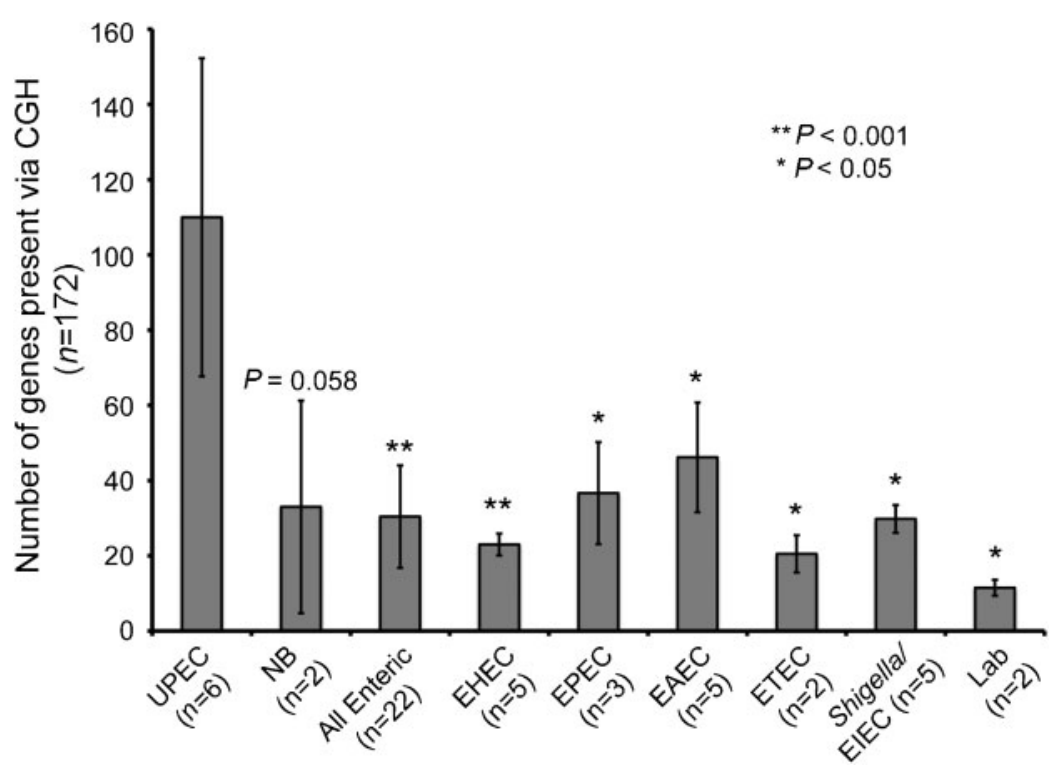

Fig. 2. Identification of UPEC virulence factors in the comparative genomic hybridization $(\mathrm{CGH})$ data. Gene features previously identified as UPEC virulence factors (Supplementary Table S3) from the CGH data were identified and examined. Groups of isolates from each of the pathovars and species were examined as labelled in Table 2. The number of virulence genes present in each of the pathovars is represented by the mean and standard deviation (or standard error). When compared with the genes present in UPEC isolates each of the other pathovars has significantly fewer UPEC virulence genes, as measured with a Student's $t$-test, with the exception of the neobladder isolates $(P=0.058)$.

\section{Distribution of the features among the $\mathbf{3 0}$ isolates}

Examination of the distribution of the number of features present or absent in each genome identifies the open pangenome nature of E. coli (Rasko et al., 2008) (Fig. 1). As mentioned previously, of the 23090 features on the array, 4471 features were present in all and 1305 features absent in all the 30 genomes examined. The remaining features are distributed among the other isolates in a pattern that suggests uninhibited lateral gene transfer between all of the pathovars and isolates. Detailed examination of each of the cluster-specific features provides genomic content that is exclusive to each of the pathogenic clusters. There are 273 features that are exclusively present among the UPEC isolates screened and not present in any of the diarrhoeagenic isolates or the neobladder isolates (see Supplementary Table S4). Many of these features are from the $E$. coli CFT073 genome (Welch et al., 2002), a prototype UPEC isolate (Mobley et al., 1990). Interestingly, there are no features that are exclusively shared between the neobladder isolates and the UPEC isolates; however, there are 154 features that are shared exclusively between the neobladder isolates and the non-UPEC isolates included in this study (see Supplementary Table S5). These data further support the hypothesis that the neobladder isolates are more similar to diarrhoeagenic isolates than to UPEC isolates.

\section{Hybridization features exclusive to the neobladder isolates}

The identification of features that were exclusive to the neobladder isolates provides insight into what may facilitate colonization of the neobladder. Genes that were exclusive to each of the neobladder strains included 100 genes for NB001 and 20 genes for NB002 (Tables 3 and 4 respectively). Nine features were identified that were exclusively present in both neobladder isolates (Table 5). All nine of these features are similar to those encoded on the ETEC plasmid pCOO (Froehlich et al., 2005). These genes are all from a type IV secretion apparatus known as the R64 thin pilus, which is utilized in liquid-based conjugations (Kim \& Komano, 1997). Based on these hybridization data, it was postulated that each isolate contains one or more plasmids.

\section{Plasmid isolation and sequencing from neobladder isolates}

The plasmids from each neobladder isolate were purified and their size examined based on gel electrophoresis (Fig. $3 \mathrm{a})$. Each of the isolates contained a large $(>50 \mathrm{~kb})$ plasmid and NB001 also contained a much smaller plasmid $(\sim 3 \mathrm{~kb})$. The plasmid preparations were then sequenced to identify if the plasmids were similar to other plasmids that have been previously identified from enteric sources. The assembled contigs for each plasmid preparation were compared with a local database containing the complete plasmids in GenBank from E. coli, Shigella and Salmonella isolates. Each isolate contained a plasmid similar to a different large plasmid. The 87 plasmid contigs from the isolate NB001, totalling $\sim 130 \mathrm{~kb}$, were similar to the pAPEC-O2-ColV plasmid (GenBank accession number AY545598; Skyberg et al., 2006). The 50 plasmid contigs, totalling $\sim 72 \mathrm{~kb}$, obtained from the NB002 isolate were most similar to the pSL476_91 plasmid from Salmonella enterica subsp. enterica serovar Heidelberg str. SL476 (GenBank accession number CP001118). For each large plasmid, the sequences do not appear to be identical to the closest relative. However, between neobladder plasmids, a conserved backbone of plasmid sequence $(41 \mathrm{~kb})$ was identified by the Mugsy alignment (Fig. 3b, c). This region contained conserved plasmid functions including replication and transfer. 
Table 3. Genes specific to isolate NB001

\begin{tabular}{|c|c|c|c|}
\hline Affy_ID & Origin & $\begin{array}{c}\text { Gene } \\
\text { designation }\end{array}$ & Annotation \\
\hline APECO1_3532_at & E. coli APECO1 & APECO1_3532 & Putative superfamily I DNA helicase \\
\hline APECO1_3530_at & E. coli APECO1 & APECO1_3530 & tia; Tia invasion determinant \\
\hline APECO1_3484_at & E. coli APECO1 & APECO1_3484 & Hypothetical protein \\
\hline APECO1_3483_at & E. coli APECO1 & APECO1_3483 & Conserved hypothetical protein; putative EAL domain \\
\hline N5007_x_at & E. coli $\mathrm{E} 2348 / 69$ & N5007 & traX; conjugal transfer pilus acetylation protein TraX \\
\hline N5010_at & E. coli $\mathrm{E} 2348 / 69$ & N5010 & traD; conjugal transfer protein $\mathrm{TraD}$ \\
\hline N5020_s_at & E. coli $\mathrm{E} 2348 / 69$ & N5020 & artA; hypothetical protein \\
\hline N5021_s_at & E. coli $\mathrm{E} 2348 / 69$ & N5021 & Hypothetical protein \\
\hline V2175_at & E. coli 7122 O78 & V2175 & Hypothetical protein \\
\hline V2176_s_at & $\begin{array}{l}\text { E. coli A2363 plasmid } \\
\text { pAPEC-O2-Col-ColV }\end{array}$ & O2ColV64 & $\begin{array}{l}\text { cvaB; colicin V secretion/processing ATP-binding protein CvaB } \\
\text { [EC 3.4.22.-] }\end{array}$ \\
\hline V2177_s_at & $\begin{array}{l}\text { E. coli A2363 plasmid } \\
\text { pAPEC-O2-Col-ColV }\end{array}$ & O2ColV63 & cvaA; colicin V secretion protein CvaA; K02022 \\
\hline V2182_s_at & $\begin{array}{l}\text { E. coli A2363 plasmid } \\
\text { pAPEC-O2-Col-ColV }\end{array}$ & O2ColV59 & $\begin{array}{l}\text { Phospho-2-dehydro-3-deoxyheptonate aldolase [EC 2.5.1.54]; } \\
\text { K01626 3-deoxy-7-phosphoheptulonate synthase [EC 2.5.1.54] }\end{array}$ \\
\hline V2189_s_at & E. coli $7122 \mathrm{O} 78$ & V2189 & Hypothetical protein \\
\hline V2198_at & E. coli $7122 \mathrm{O} 78$ & V2198 & Hypothetical protein \\
\hline V2200_at & E. coli 7122 O78 & V2200 & Hypothetical protein \\
\hline V2202_s_at & $\begin{array}{l}\text { E. coli A2363 plasmid } \\
\text { pAPEC-O2-Col-ColV }\end{array}$ & O2ColV34 & $\begin{array}{l}\text { etsB; EtsB; K05685 macrolide transport system permease } \\
\text { protein; K05686 macrolide transport system ATP-binding } \\
\text { protein }\end{array}$ \\
\hline O2Colv33_s_at & $\begin{array}{l}\text { E. coli A2363 plasmid } \\
\text { pAPEC-O2-Col-ColV }\end{array}$ & O2Colv32 & etsA; putative type I secretion membrane-fusion protein EtsA \\
\hline V2205_at & E. coli 7122 O78 & V2205 & Hypothetical protein \\
\hline V2206_at & E. coli $7122 \mathrm{O} 78$ & V2206 & Hypothetical protein \\
\hline V2213_s_at & E. coli plasmid p1658/97 & p166897_107 & Mig-14 family protein \\
\hline V2224_s_at & $\begin{array}{l}\text { E. coli A2363 plasmid } \\
\text { pAPEC-O2-Col-ColV }\end{array}$ & O2ColV4 & $\begin{array}{l}\text { sitD; SitD protein, iron transport protein, inner membrane } \\
\text { component }\end{array}$ \\
\hline V2227_s_at & $\begin{array}{l}\text { E. coli A2363 plasmid } \\
\text { pAPEC-O2-Col-ColV }\end{array}$ & O2ColV11 & iucA; aerobactin siderophore biosynthesis protein IucA \\
\hline V2233_s_at & $\begin{array}{l}\text { E. coli A2363 plasmid } \\
\text { pAPEC-O2-Col-ColV }\end{array}$ & O2ColV14 & iucD; L-lysine 6-monooxigenase IucD [EC 1.14.13.59] \\
\hline V2234_s_at & $\begin{array}{l}\text { E. coli A2363 plasmid } \\
\text { pAPEC-O2-Col-ColV }\end{array}$ & O2ColV15 & iutA; ferric aerobactin receptor precursor \\
\hline IS2_03_s_at & E. coli K-12 MG1655 & IS2_3 & $\begin{array}{l}\text { aph, kan; aminoglycoside } 3^{\prime} \text {-phosphotransferase; K00897 } \\
\text { aminoglycoside } 3^{\prime} \text {-phosphotransferase [EC 2.7.1.95] }\end{array}$ \\
\hline p166897_027_at & E. coli plasmid p1658/97 & p166897_027 & artA; hypothetical protein \\
\hline p166897_029_x_at & E. coli plasmid p1658/97 & p166897_029 & Putative conjugal transfer protein TrbB \\
\hline p166897_031_s_at & E. coli plasmid p1658/97 & p166897_031 & trbF; conjugal transfer protein TrbF \\
\hline p166897_106_at & E. coli plasmid p1658/97 & p166897_106 & hlyF; haemolysin HlyF \\
\hline pIGAL1_01_s_at & E. coli A2363 plasmid pAPEC-O2-R & O2R_122 & Transposase \\
\hline pIGAL1_02_s_at & E. coli A2363 plasmid pAPEC-O2-R & O2R_123 & Transposon $\operatorname{Tn} 3$ resolvase \\
\hline O2R_06_at & E. coli A2363 plasmid pAPEC-O2-R & O2R_6 & Hypothetical protein \\
\hline O2R_24_x_at & $\begin{array}{l}\text { E. coli A2363 plasmid } \\
\text { pAPEC-O2-Col-ColV }\end{array}$ & O2ColV118 & traQ; conjugal transfer pilin chaperone TraQ \\
\hline LH0096_x_at & E. coli $\mathrm{B} 171$ & LH0096 & Conserved predicted protein \\
\hline LH0129_at & E. coli $\mathrm{B} 171$ & LH0129 & Putative immunity protein for colicin IA \\
\hline pO86A1_p048_x_at & E. coli plasmid pO861A1 & pO86A1_p048 & imm; colicin-Ia immunity protein \\
\hline O2ColV164_at & $\begin{array}{l}\text { E. coli A2363 plasmid } \\
\text { pAPEC-O2-Col-ColV }\end{array}$ & O2ColV164 & Hypothetical protein \\
\hline O2ColV168_at & $\begin{array}{l}\text { E. coli A2363 plasmid } \\
\text { pAPEC-O2-Col-ColV }\end{array}$ & O2ColV168 & Hypothetical protein \\
\hline O2ColV169_at & $\begin{array}{l}\text { E. coli A2363 plasmid } \\
\text { pAPEC-O2-Col-ColV }\end{array}$ & O2Colv169 & Hypothetical protein \\
\hline
\end{tabular}


Table 3. cont.

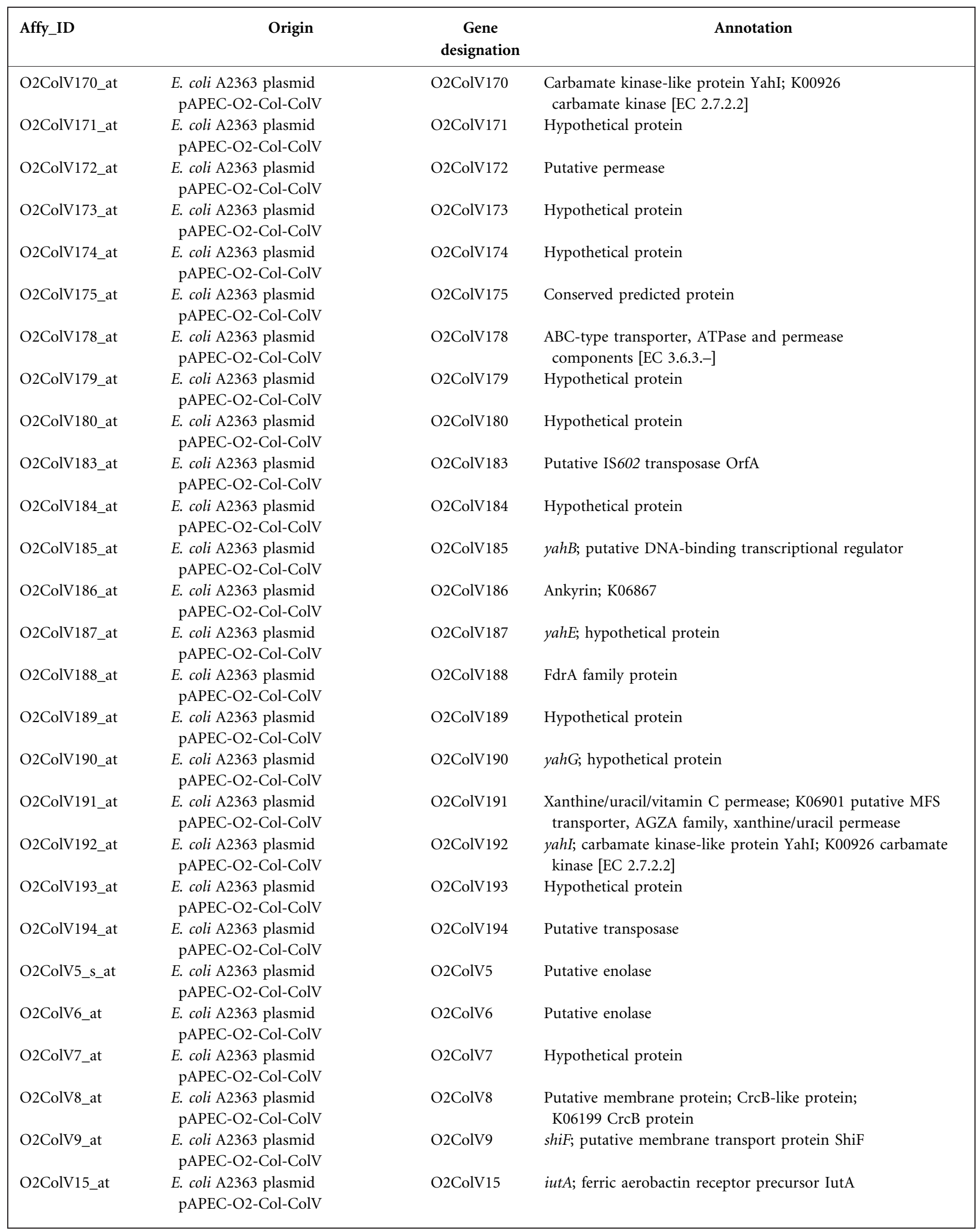


Table 3. cont.

\begin{tabular}{|c|c|c|c|}
\hline Affy_ID & Origin & $\begin{array}{c}\text { Gene } \\
\text { designation }\end{array}$ & Annotation \\
\hline O2ColV16_at & $\begin{array}{l}\text { E. coli A2363 plasmid } \\
\text { pAPEC-O2-Col-ColV }\end{array}$ & O2ColV16 & Hypothetical protein \\
\hline O2ColV21_at & $\begin{array}{l}\text { E. coli A2363 plasmid } \\
\text { pAPEC-O2-Col-ColV }\end{array}$ & O2ColV21 & Hypothetical protein \\
\hline O2ColV25_at & $\begin{array}{l}\text { E. coli A2363 plasmid } \\
\text { pAPEC-O2-Col-ColV }\end{array}$ & O2ColV25 & Hypothetical protein \\
\hline O2ColV26_at & $\begin{array}{l}\text { E. coli A2363 plasmid } \\
\text { pAPEC-O2-Col-ColV }\end{array}$ & O2Colv26 & ompT; outer membrane protease [EC 3.4.23.49] \\
\hline O2ColV27_at & $\begin{array}{l}\text { E. coli A2363 plasmid } \\
\text { pAPEC-O2-Col-ColV }\end{array}$ & O2ColV27 & Hypothetical protein \\
\hline O2ColV28_at & $\begin{array}{l}\text { E. coli A2363 plasmid } \\
\text { pAPEC-O2-Col-ColV }\end{array}$ & O2ColV28 & Hypothetical protein \\
\hline O2ColV29_at & $\begin{array}{l}\text { E. coli A2363 plasmid } \\
\text { pAPEC-O2-Col-ColV }\end{array}$ & O2ColV29 & Transposase B; K07497 putative transposase \\
\hline O2ColV46_at & $\begin{array}{l}\text { E. coli A2363 plasmid } \\
\text { pAPEC-O2-Col-ColV }\end{array}$ & O2ColV46 & Putative transposase ORF B (fragment), IS2 \\
\hline O2ColV47_s_at & $\begin{array}{l}\text { E. coli A2363 plasmid } \\
\text { pAPEC-O2-Col-ColV }\end{array}$ & O2ColV47 & Conserved hypothetical protein; putative GTPase \\
\hline O2ColV49_x_at & $\begin{array}{l}\text { E. coli A2363 plasmid } \\
\text { pAPEC-O2-Col-ColV }\end{array}$ & O2ColV49 & borD; putative lipoprotein; DLP12 prophage \\
\hline O2ColV49.2_at & $\begin{array}{l}\text { E. coli A2363 plasmid } \\
\text { pAPEC-O2-Col-ColV }\end{array}$ & O2ColV49.2 & Hypothetical protein \\
\hline O2ColV51_x_at & $\begin{array}{l}\text { E. coli A2363 plasmid } \\
\text { pAPEC-O2-Col-ColV }\end{array}$ & O2ColV51 & Predicted transposase \\
\hline O2ColV58_at & $\begin{array}{l}\text { E. coli A2363 plasmid } \\
\text { pAPEC-O2-Col-ColV }\end{array}$ & O2ColV58 & Hypothetical protein \\
\hline O2ColV60_at & $\begin{array}{l}\text { E. coli A2363 plasmid } \\
\text { pAPEC-O2-Col-ColV }\end{array}$ & O2ColV60 & Hypothetical protein \\
\hline O2ColV65_at & $\begin{array}{l}\text { E. coli A2363 plasmid } \\
\text { pAPEC-O2-Col-ColV }\end{array}$ & O2ColV65 & cvaC; colicin $\mathrm{V}$ precursor (microcin $\mathrm{V}$ bacteriocin) \\
\hline O2ColV66_at & $\begin{array}{l}\text { E. coli A2363 plasmid } \\
\text { pAPEC-O2-Col-ColV }\end{array}$ & O2ColV66 & $c v i$; colicin $\mathrm{V}$ immunity protein \\
\hline O2ColV67_at & $\begin{array}{l}\text { E. coli A2363 plasmid } \\
\text { pAPEC-O2-Col-ColV }\end{array}$ & O2ColV67 & Hypothetical protein \\
\hline O2ColV68_s_at & $\begin{array}{l}\text { E. coli A2363 plasmid } \\
\text { pAPEC-O2-Col-ColV }\end{array}$ & O2ColV68 & Hypothetical protein \\
\hline O2ColV75_at & $\begin{array}{l}\text { E. coli A2363 plasmid } \\
\text { pAPEC-O2-Col-ColV }\end{array}$ & O2ColV75 & Resolvase domain-containing protein \\
\hline O2ColV76_at & $\begin{array}{l}\text { E. coli A2363 plasmid } \\
\text { pAPEC-O2-Col-ColV }\end{array}$ & O2ColV76 & Hypothetical protein \\
\hline O2ColV78_at & $\begin{array}{l}\text { E. coli A2363 plasmid } \\
\text { pAPEC-O2-Col-ColV }\end{array}$ & O2ColV78 & DNA-binding protein \\
\hline O2ColV84_at & $\begin{array}{l}\text { E. coli A2363 plasmid } \\
\text { pAPEC-O2-Col-ColV }\end{array}$ & O2ColV84 & Resolvase \\
\hline O2ColV85_at & $\begin{array}{l}\text { E. coli A2363 plasmid } \\
\text { pAPEC-O2-Col-ColV }\end{array}$ & O2ColV85 & Resolvase \\
\hline O2ColV91_at & $\begin{array}{l}\text { E. coli A2363 plasmid } \\
\text { pAPEC-O2-Col-ColV }\end{array}$ & O2ColV91 & $k d a$; putative klebicin $\mathrm{D}$ activity protein \\
\hline pCool11_at & E. coli plasmid pCoo & pCool11 & Prepilin peptidase \\
\hline pCool12_at & E. coli plasmid pCoo & pCoo112 & Lytic transglycosylase PilT \\
\hline pCool13_at & E. coli plasmid pCoo & pCool13 & Type IV prepilin \\
\hline pCool14_at & E. coli plasmid pCoo & pCool14 & Integral membrane protein \\
\hline pCool16_at & E. coli plasmid pCoo & pCool16 & pilP; type IV pilus biogenesis protein PilP \\
\hline pCool18_at & E. coli plasmid pCoo & pCool18 & $\begin{array}{l}\text { Type IVB pilus formation outer-membrane protein, } \\
\text { R64 PilN family }\end{array}$ \\
\hline
\end{tabular}


Table 3. cont.

\begin{tabular}{|llll|}
\hline Affy_ID & Origin & $\begin{array}{c}\text { Gene } \\
\text { designation }\end{array}$ & Annotation \\
\hline pCoo121_at & E. coli plasmid pCoo & pCoo121 & PilK protein \\
pCoo122_at & E. coli plasmid pCoo & pCoo122 & PilJ protein \\
pCoo123_at & E. coli plasmid pCoo & pCoo123 & Pill protein \\
pCoo125_at & E. coli plasmid pCoo & pCool25 & PilK protein \\
\hline
\end{tabular}

\section{Screening of environmental, diarrhoeagenic and uropathogenic $E$. coli for features identified in neobladder isolates}

Gene features were selected for PCR screening from the CGH data that were present in both of the neobladder isolates and not in any other group (three features), exclusive to each of the neobladder isolates (three features for NB001, two features for NB002) or more broadly conserved in UPEC (two features) or enteric E. coli isolates (three features) (Supplementary Fig. S1). Primer sequences and gene features are listed in Supplementary Table S1. We interrogated 133 E. coli isolates: 61 isolates from environmental sources or non-human animals (http://www.shigatox.net/stec/cgi-bin/ index), 21 diarrhoeagenic isolates (http://www.shigatox.net/ stec/cgi-bin/index) and 51 isolates from human urinary tracts (Mobley et al., 1990). Gene features that were demonstrated in the CGH data to be more prevalent in the UPEC or enteric genomes were more prevalent in each respective sample population when examined by PCR screening in the larger study group. For example, genes identified as more common in UPEC isolates by CGH were more prevalent in the examined UPEC isolates than in the enteric or environmental isolates (Fig. 4). The features that were identified in one or more of the neobladder isolates were not widely distributed among

Table 4. Genes specific to isolate NB002

\begin{tabular}{|c|c|c|c|c|}
\hline Affy_ID & Origin & GenBank accession & Gene designation & Annotation \\
\hline SFV_0202_at & $\begin{array}{l}\text { Shigella flexneri } \\
5 \text { str. } 8401\end{array}$ & NC_008258 & SFV_0202 & $\begin{array}{l}\text { Hypothetical protein; K11895 type VI secretion system } \\
\text { protein ImpH }\end{array}$ \\
\hline SFV_0630_at & $\begin{array}{l}\text { Shigella flexneri } \\
5 \text { str. } 8401\end{array}$ & NC_008258 & SFV_0630 & Putative $r h s$-family protein \\
\hline SD1_0080_x_at & Shigella sp. & AAMJ01000001.1 & SD1_0080 & $\begin{array}{l}\text { Putative L-xylulose-5-phosphate 3-epimerase; K03082 } \\
\text { hexulose-6-phosphate isomerase [EC 5.-.-.-] }\end{array}$ \\
\hline SD1_0822_at & Shigella sp. & AAMJ01000002.1 & SD1_0822 & Hypothetical protein \\
\hline SD1_0823_at & Shigella sp. & AAMJ01000002.1 & SD1_0823 & Transcriptional regulator \\
\hline SD1_0824_at & Shigella sp. & AAMJ01000002.1 & SD1_0824 & $\begin{array}{l}\text { Galactitol-specific enzyme IIA of phosphotransferase } \\
\text { system; K02773 PTS system, galactitol-specific IIA } \\
\text { component [EC 2.7.1.69] }\end{array}$ \\
\hline SD1_0827_at & Shigella sp. & AAMJ01000002.1 & SD1_0827 & PTS system, galactitol-specific IIC component \\
\hline SD1_3295_at & Shigella sp. & AAMJ01000019.1 & SD1_3295 & $\begin{array}{l}\text { Hypothetical protein; K11891 type VI secretion system } \\
\text { protein ImpL }\end{array}$ \\
\hline N0356_x_at & E. coli $\mathrm{E} 2348 / 69$ & E2348_69000001.1 & N0356 & Hypothetical protein \\
\hline N0946_at & E. coli $\mathrm{E} 2348 / 69$ & E2348_69000001.1 & N0946 & Hypothetical protein \\
\hline N0948_at & E. coli $\mathrm{E} 2348 / 69$ & E2348_69000001.1 & N0948 & Hypothetical protein \\
\hline N0950_at & E. coli $\mathrm{E} 2348 / 69$ & E2348_69000001.1 & N0950 & Hypothetical protein \\
\hline N0953_at & E. coli E2348/69 & E2348_69000001.1 & N0953 & Hypothetical protein \\
\hline N1524_at & E. coli $\mathrm{E} 2348 / 69$ & E2348_69000001.1 & N1524 & $p d u F$; propanediol diffusion facilitator \\
\hline N1528_at & E. coli E2348/69 & E2348_69000001.1 & N1528 & Hypothetical protein \\
\hline N1529_at & E. coli E2348/69 & E2348_69000001.1 & N1529 & Hypothetical protein \\
\hline
\end{tabular}


Table 5. Genes identified by $\mathrm{CGH}$ as only present in the neobladder isolates

\begin{tabular}{|llllll|}
\hline Affy_ID & \multicolumn{1}{c}{ Origin } & $\begin{array}{c}\text { GenBank } \\
\text { accession }\end{array}$ & $\begin{array}{c}\text { Gene } \\
\text { number }\end{array}$ & $\begin{array}{l}\text { Gene } \\
\text { name }\end{array}$ \\
\hline pCoo111_at & E. coli plasmid pCoo & NC_007635 & pCool11 & pilU & Prepilin peptidase \\
pCoo112_at & E. coli plasmid pCoo & NC_007635 & pCool12 & pilT & Putative transglycosylase PilT \\
pCoo113_at & E. coli plasmid pCoo & NC_007635 & pCoo113 & pils & Type IV prepilin \\
pCoo114_at & E. coli plasmid pCoo & NC_007635 & pCoo114 & pilR & Integral membrane protein \\
pCoo116_at & E. coli plasmid pCoo & NC_007635 & pCoo116 & pilP & PilP protein \\
pCoo118_at & E. coli plasmid pCoo & NC_007635 & pCoo118 & pilN & PilN protein \\
pCoo121_at & E. coli plasmid pCoo & NC_007635 & pCoo121 & pilK & PilK protein \\
pCoo122_at & E. coli plasmid pCoo & NC_007635 & pCoo122 & pilJ & PilJ protein \\
pCoo123_at & E. coli plasmid pCoo & NC_007635 & pCool23 & pill & Pill protein \\
\hline
\end{tabular}

the isolates of the three populations examined and did not appear to correlate based on the origin of the isolates.

\section{DISCUSSION}

Previous studies of neobladder-associated bacteria have focused on a limited number of virulence factors (Keegan et al., 2000, 2003), but to our knowledge this is the first study of neobladder isolates on a genomic scale. In this study, the genomic content of two E. coli isolates obtained from individuals who had had neobladder surgery were examined. Comparative genomic hybridization demonstrated that the neobladder isolates were not in the same cluster as UPEC isolates, based on presence/absence in the variable gene content, but were more similar to the diarrhoeagenic, commensal or laboratory E. coli isolates. The distribution of gene presence and absence among the collection of 30 isolates examined using a pan-E. coli/Shigella genome microarray suggested that there was no barrier to horizontal gene transfer. These findings were extended by examining the hybridization patterns of known UPEC virulence factors, and these data indicate that the neobladder isolates are selected for by the environment from which they were isolated much in the same way that UPEC isolates are selected by the environment of the urinary tract (Chen et al.,
2006, 2009). The five UPEC isolates examined in this study exclusively shared 273 features (see Supplementary Table S4), many originating in UPEC isolates or the closely related avian E. coli (Johnson et al., 2007). This suggests that there is a conserved gene repertoire selected among the UPEC isolates for survival and/or colonization of the urinary tract. Interestingly, no array feature was shared exclusively between the UPEC and the neobladder isolates, whereas 154 features were shared with the neobladder, diarrhoeagenic, commensal or laboratory-adapted isolates and not the UPECs (Supplementary Table S5). Further screening by PCR of a collection of $133 \mathrm{E}$. coli isolates indicated that the genes unique to the neobladder isolates were not common among the environmental, diarrhoeagenic or UPEC isolates screened. This suggests that while on a genomic scale the neobladder isolates are similar to enteric isolates, there does appear to be some unknown pressure to retain the neobladder-specific genes that are not observed in the other populations. These analyses indicate that the tropism of the bacterium to the gastrointestinal tissue in a neobladder is stronger than the selective pressure of the UPEC genes to survive or colonize in the presence of urine.

One question that remains unanswered by this study is whether the neobladder isolates obtained at 8 months post surgery and over 10 years post surgery were present in
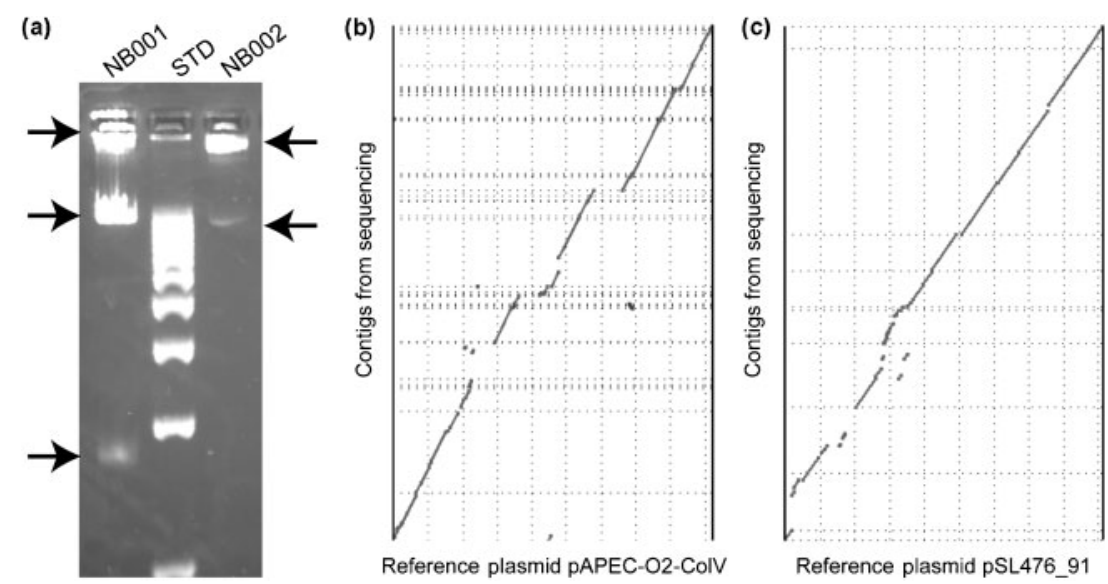

Fig. 3. Neobladder isolates contain plasmids. (a) Plasmids were isolated from overnight cultures of NB001 and NB002 using the Phase prep BAC DNA kit (Sigma NA0100$1 \mathrm{KT}$ ) plasmid preparation kit. Plasmids were visualized on a $1.0 \%$ agarose gel stained with ethidium bromide (STD, Invitrogen $1 \mathrm{~kb}$ Plus DNA Ladder 8). (b, c) MUMmer plots demonstrating that in selected sequencing the plasmids are most similar to pAPEC-O2-ColV and pSL476-91 in NB001 and NB002 respectively. The compared plasmids demonstrate coverage of the entire plasmid and are $>180$ and $90 \mathrm{~kb}$ respectively. 


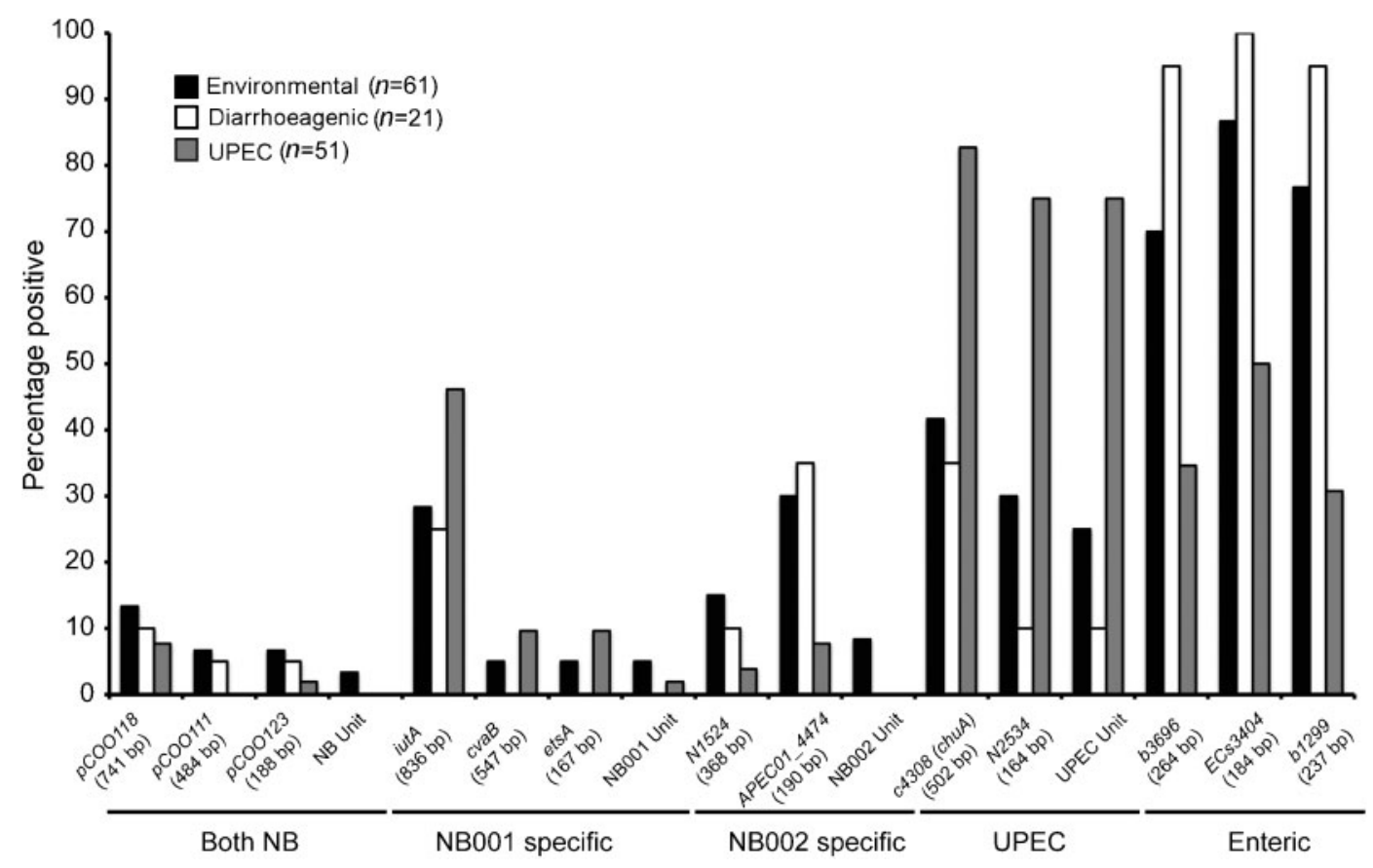

Fig. 4. PCR screening of environmental, diarrhoeagenic and uropathogenic E. coli. A total of 133 isolates were selected for PCR-based screening representing populations from environmental (black bars), diarrhoeagenic (white bars) and uropathogenic (grey bars) sources. Assays were developed which represented genes that, according to $\mathrm{CGH}$, were conserved in both neobladder isolates, either neobladder isolate, UPEC or enteric isolates. The neobladder features were present only in few isolates from any of the populations (first three groups of assays). When taken as a unit, these genes were present in even fewer isolates. UPEC and enteric gene features were present in the UPEC or diarrhoeagenic/environmental isolates, respectively. These data suggest that the neobladder-specific genes are not widely distributed in these populations.

association with the tissue as it was transported from the ileum to the urinary tract. Antibiotics are frequently employed prior to and after surgery to decrease the bacterial load. Additionally, there is a process of physical cleaning of the ileal tissue to be used for neobladder construction; however, this tissue can never be made sterile and there is the likely carryover of microbiota that includes E. coli (Studer et al., 1991, 1996). It is possible that the microbiota, and E. coli as a member of the gastrointestinal flora, were transferred from the gastrointestinal tract to the urinary tract with the tissue. The E. coli isolate obtained over 10 years post surgery is unlikely to be identical to an original isolate from the gastrointestinal tract, but the isolate obtained less than 1 year post surgery is a possible remnant of the gastrointestinal tract flora. Another remaining question is whether the isolates obtained are representative of the E. coli in the neobladder community over time; for example, if isolates were examined longitudinally would we find the same isolate multiple times or are the isolates in flux over time? This study represents a preliminary foray into the genomics of $E$. coli neobladder isolates and does not suggest that this limited number of isolates represents the complete genetic diversity of neobladder isolates. Further study of larger sample collections, including additional neobladder isolates, will be required to determine if these findings are generally applicable to a large collection of neobladder E. coli.
The comparison of multiple isolates from each of the major pathovars helps to identify how each of these isolates are related on a genomic scale (Fig. 1). Recent genomic sequencing studies have examined multiple genomes and demonstrated that there is a significant amount of variability within each of the E. coli pathovars (Rasko et al., 2008; Touchon et al., 2009). Comparative genomic hybridization using a pan-genome array allows for rapid and accurate examination of the genomic content and provides a limited view of the genetic variability and horizontal gene transfer between the isolates in relation to the data that are contained on the microarray. Interestingly, the relative groupings in Fig. 1 are similar to the phylogenetic relationships that have been previously determined for a number of these isolates (Donnenberg \& Whittam, 2001; Whittam et al., 1983, 1993). In this study, a number of interesting phylogenetic trends are observed that must be confirmed with larger strain collections. For example, the EAEC isolates examined do not consistently fall into any one of the clusters observed in this study. This is congruent with MLST studies suggesting that this pathovar was more variable than other pathovars in terms of phylogeny (Czeczulin et al., 1999; Valentiner-Branth et al., 2003) and in the ability to cause disease in humans (Nataro et al., 1995). EHEC was the one other pathovar that was represented in multiple clusters; however, this observation 
is consistent with the literature on EHEC evolution based on MLST and virulence markers (Donnenberg \& Whittam, 2001). The O157:H7 isolates EDL933, 86-24 and Sakai are members of the EHEC1 group whereas OK1114 and TX1999 are recent $\mathrm{O} 111: \mathrm{H} 8$ isolates and members of the EHEC2 phylogenetic group (Donnenberg \& Whittam, 2001). The comparative genomic hybridization and analysis confirm general MLST and virulence gene typing but provide a much more robust method to examine these isolates on a genomic scale. By continuing to expand our bank of genomic hybridizations, we can rapidly and accurately examine and include new isolates in these comparative studies.

In addition to comparing the hybridization profiles to a bank of reference genome hybridizations, the information from the hybridization of these neobladder isolates was also used to focus further analysis on the plasmid content. The hybridization patterns of the unique features of the neobladder isolates (Table 5) suggested that each isolate contained at least one plasmid. The genes shared only between the neobladder isolates are part of a type IV secretion system from the pCOO plasmid in ETEC (Froehlich et al., 2005). Other genes that are specific to each of the isolates (Tables 3 and 4) are also part of plasmid transfer or replication genes, suggesting that the core of the type IV system is similar but not identical between these two plasmids. Further isolation and sequencing of the plasmids from each isolate revealed that each isolate harboured a large plasmid (>50 kb, Fig. 3). The NB001 plasmid was most similar to the avian pathogenic E. coli (APEC) plasmid (Johnson et al., 2007) and the NB002 plasmid was most similar to the Salmonella enterica subsp. enterica serovar Heidelberg str. SL476 pSL476_91 plasmid. Each of these plasmids are common mobile plasmids in the enteric pathogens (Johnson \& Nolan, 2009) and further represent the horizontal gene transfer of these isolates and their similarity to the E. coli from the gastrointestinal tract.

Patients with neobladders often indicate that it is difficult to determine if they are experiencing a urinary tract infection, as the symptomology does not present in the same manner as a typical UTI, even though the bacterial counts may be clinically significant (Suriano et al., 2008; Wullt et al., 2004). There is often an absence of pain, and the incidence of upper urinary tract involvement is less common than in traditional UTI (Kristjánsson et al., 1995a, b). This lack of ascending infection is often attributed to the fact that the neobladder is a low-pressure vesicle and thus there is a lack of pressure to aid the bacterium in ascending the ureters. However, genomic examination of neobladder isolates indicates that these isolates are more similar to the diarrhoeagenic isolates and not similar to UPEC isolates. It is possible that the E. coli isolates within a neobladder, being more similar to diarrhoeagenic, commensal and laboratory-adapted isolates, do not have the genetic capability to ascend the ureters, adhere to proximal tubule epithelium and cause pyelonephritis or subsequent systemic infection. Additionally, it may be that colonization by a commensal-like E. coli that causes no disease ends up creating a 'probiotic effect' that inhibits colonization by UPEC-like strains, thus preventing the secondary disease often observed. The ability of the gastrointestinal tissue to tolerate interactions with bacterial communities may prove beneficial, as colonized tissue does not result in a symptomatic presentation. This study allows us to postulate that the 'colonization' of the neobladder could, and most likely should, be considered a commensal colonization of gastrointestinal tissue and not an infectious process requiring treatment.

\section{ACKNOWLEDGEMENTS}

This work was supported from startup funds from the State of Maryland to the Rasko laboratory.

\section{REFERENCES}

Åkerlund, S., Campanello, M., Kaijser, B. \& Jonsson, O. (1994). Bacteriuria in patients with a continent ileal reservoir for urinary diversion does not regularly require antibiotic treatment. Br J Urol 74, 177-181.

Altschul, S. F., Gish, W., Miller, W., Myers, E. W. \& Lipman, D. J. (1990). Basic local alignment search tool. J Mol Biol 215, 403410.

Angiuoli, S. V. \& Salzberg, S. L. (2011). Mugsy: fast multiple alignment of closely related whole genomes. Bioinformatics 27, 334342.

Blattner, F. R., Plunkett, G., III, Bloch, C. A., Perna, N. T., Burland, V., Riley, M., Collado-Vides, J., Glasner, J. D., Rode, C. K. \& other authors (1997). The complete genome sequence of Escherichia coli K-12. Science 277, 1453-1462.

Chen, S. L., Hung, C. S., Xu, J., Reigstad, C. S., Magrini, V., Sabo, A., Blasiar, D., Bieri, T., Meyer, R. R. \& other authors (2006). Identification of genes subject to positive selection in uropathogenic strains of Escherichia coli: a comparative genomics approach. Proc Natl Acad Sci U S A 103, 5977-5982.

Chen, S. L., Hung, C. S., Pinkner, J. S., Walker, J. N., Cusumano, C. K., Li, Z., Bouckaert, J., Gordon, J. I. \& Hultgren, S. J. (2009). Positive selection identifies an in vivo role for FimH during urinary tract infection in addition to mannose binding. Proc Natl Acad Sci U S A 106, 22439-22444.

Czeczulin, J. R., Whittam, T. S., Henderson, I. R., Navarro-Garcia, F. \& Nataro, J. P. (1999). Phylogenetic analysis of enteroaggregative and diffusely adherent Escherichia coli. Infect Immun 67, 2692-2699.

Davidsson, T., Wullt, B., Könyves, J., Månsson, A. \& Månsson, W. (2000). Urinary diversion and bladder substitution in patients with bladder cancer. Urol Oncol 5, 224-231.

Delcher, A. L., Phillippy, A., Carlton, J. \& Salzberg, S. L. (2002). Fast algorithms for large-scale genome alignment and comparison. Nucleic Acids Res 30, 2478-2483.

Delcher, A. L., Salzberg, S. L. \& Phillippy, A. M. (2003). Using MUMmer to identify similar regions in large sequence sets. Curr Protoc Bioinformatics Chapter 10, 10.3.

Donnenberg, M. S. \& Whittam, T. S. (2001). Pathogenesis and evolution of virulence in enteropathogenic and enterohemorrhagic Escherichia coli. J Clin Invest 107, 539-548.

Falagas, M. E. \& Vergidis, P. I. (2005). Urinary tract infections in patients with urinary diversion. Am J Kidney Dis 46, 1030-1037. 
Fang, H., Xu, J., Ding, D., Jackson, S. A., Patel, I. R., Frye, J. G., Zou, W., Nayak, R., Foley, S. \& other authors (2010). An FDA bioinformatics tool for microbial genomics research on molecular characterization of bacterial foodborne pathogens using microarrays. BMC Bioinformatics 11 (Suppl 6), S4.

Froehlich, B., Parkhill, J., Sanders, M., Quail, M. A. \& Scott, J. R. (2005). The pCoo plasmid of enterotoxigenic Escherichia coli is a mosaic cointegrate. J Bacteriol 187, 6509-6516.

Fukiya, S., Mizoguchi, H., Tobe, T. \& Mori, H. (2004). Extensive genomic diversity in pathogenic Escherichia coli and Shigella strains revealed by comparative genomic hybridization microarray. J Bacteriol 186, 3911-3921.

Ge, Z. \& Taylor, D. E. (1992). H. pylori DNA transformation by natural competence and electroporation. In Helicobacter pylori Protocols, pp. 145-152. Edited by C. L. Clayton \& H. L. T. Mobley. Totowa: Humana Press.

Hale, T. L., Guerry, P., Seid, R. C., Jr, Kapfer, C., Wingfield, M. E., Reaves, C. B., Baron, L. S. \& Formal, S. B. (1984). Expression of lipopolysaccharide $\mathrm{O}$ antigen in Escherichia coli K-12 hybrids containing plasmid and chromosomal genes from Shigella dysenteriae 1. Infect Immun 46, 470-475.

Hayashi, T., Makino, K., Ohnishi, M., Kurokawa, K., Ishii, K., Yokoyama, K., Han, C. G., Ohtsubo, E., Nakayama, K. \& other authors (2001). Complete genome sequence of enterohemorrhagic Escherichia coli $\mathrm{O} 157: \mathrm{H} 7$ and genomic comparison with a laboratory strain K-12. DNA Res 8, 11-22.

Hochhut, B., Wilde, C., Balling, G., Middendorf, B., Dobrindt, U., Brzuszkiewicz, E., Gottschalk, G., Carniel, E. \& Hacker, J. (2006). Role of pathogenicity island-associated integrases in the genome plasticity of uropathogenic Escherichia coli strain 536. Mol Microbiol 61, 584-595.

Hubbell, E., Liu, W. M. \& Mei, R. (2002). Robust estimators for expression analysis. Bioinformatics 18, 1585-1592.

Iwakiri, J. \& Freiha, F. (1993). Stanford pouch ileal neobladder: clinical, radiologic, and urodynamic follow-up. Urology 41, 517-522. Iwakiri, J., Gill, H., Anderson, R. \& Freiha, F. (1993). Functional and urodynamic characteristics of an ileal neobladder. J Urol 149, 10721076.

Iwakiri, J., Freiha, F. S. \& Shortliffe, L. M. (2002). Prospective study of urinary tract infections and urinary antibodies after radical prostatectomy and cystoprostatectomy. Urol Clin North Am 29, 251-258, xii.

Johnson, T. J. \& Nolan, L. K. (2009). Pathogenomics of the virulence plasmids of Escherichia coli. Microbiol Mol Biol Rev 73, 750-774.

Johnson, T. J., Kariyawasam, S., Wannemuehler, Y., Mangiamele, P., Johnson, S. J., Doetkott, C., Skyberg, J. A., Lynne, A. M., Johnson, J. R. \& Nolan, L. K. (2007). The genome sequence of avian pathogenic Escherichia coli strain $\mathrm{O} 1: \mathrm{K} 1: \mathrm{H} 7$ shares strong similarities with human extraintestinal pathogenic E. coli genomes. J Bacteriol 189, 3228-3236.

Keegan, S. J., Hormaeche, C. E., Pearson, J. P. \& Gally, D. L. (2000). Characterisation and adherence mechanisms of Escherichia coli strains causing infections in patients with a reconstructed bladder. $A d v \operatorname{Exp}$ Med Biol 485, 263-271.

Keegan, S. J., Graham, C., Neal, D. E., Blum-Oehler, G., N'Dow, J., Pearson, J. P. \& Gally, D. L. (2003). Characterization of Escherichia coli strains causing urinary tract infections in patients with transposed intestinal segments. J Urol 169, 2382-2387.

Kim, S. R. \& Komano, T. (1997). The plasmid R64 thin pilus identified as a type IV pilus. J Bacteriol 179, 3594-3603.

Kristjánsson, A., Bajc, M., Wallin, L., Willner, J. \& Månsson, W. (1995a). Renal function up to 16 years after conduit (refluxing or anti-reflux anastomosis) or continent urinary diversion. 2. Renal scarring and location of bacteriuria. Br J Urol 76, 546-550.

Kristjánsson, A., Wallin, L. \& Månsson, W. (1995b). Renal function up to 16 years after conduit (refluxing or anti-reflux anastomosis) or continent urinary diversion. 1. Glomerular filtration rate and patency of uretero-intestinal anastomosis. Br J Urol 76, 539-545.

Kurtz, S., Phillippy, A., Delcher, A. L., Smoot, M., Shumway, M., Antonescu, C. \& Salzberg, S. L. (2004). Versatile and open software for comparing large genomes. Genome Biol 5, R12.

Lane, D. J., Pace, B., Olsen, G. J., Stahl, D. A., Sogin, M. L. \& Pace, N. R. (1985). Rapid determination of $16 \mathrm{~S}$ ribosomal RNA sequences for phylogenetic analyses. Proc Natl Acad Sci U S A 82, 6955-6959.

Liu, W. M., Mei, R., Di, X., Ryder, T. B., Hubbell, E., Dee, S., Webster, T. A., Harrington, C. A., Ho, M. H. \& other authors (2002). Analysis of high density expression microarrays with signed-rank call algorithms. Bioinformatics 18, 1593-1599.

Lloyd, A. L., Rasko, D. A. \& Mobley, H. L. (2007). Defining genomic islands and uropathogen-specific genes in uropathogenic Escherichia coli. J Bacteriol 189, 3532-3546.

Lloyd, A. L., Henderson, T. A., Vigil, P. D. \& Mobley, H. L. (2009). Genomic islands of uropathogenic Escherichia coli contribute to virulence. J Bacteriol 191, 3469-3481.

Mobley, H. L., Green, D. M., Trifillis, A. L., Johnson, D. E., Chippendale, G. R., Lockatell, C. V., Jones, B. D. \& Warren, J. W. (1990). Pyelonephritogenic Escherichia coli and killing of cultured human renal proximal tubular epithelial cells: role of hemolysin in some strains. Infect Immun 58, 1281-1289.

Nataro, J. P., Deng, Y., Cookson, S., Cravioto, A., Savarino, S. J., Guers, L. D., Levine, M. M. \& Tacket, C. O. (1995). Heterogeneity of enteroaggregative Escherichia coli virulence demonstrated in volunteers. J Infect Dis 171, 465-468.

Nie, H., Yang, F., Zhang, X., Yang, J., Chen, L., Wang, J., Xiong, Z., Peng, J., Sun, L. \& other authors (2006). Complete genome sequence of Shigella flexneri $5 \mathrm{~b}$ and comparison with Shigella flexneri 2a. BMC Genomics 7, 173.

Perna, N. T., Plunkett, G., III, Burland, V., Mau, B., Glasner, J. D., Rose, D. J., Mayhew, G. F., Evans, P. S., Gregor, J. \& other authors (2001). Genome sequence of enterohaemorrhagic Escherichia coli O157 : H7. Nature 409, 529-533.

Rasko, D. A., Rosovitz, M. J., Myers, G. S., Mongodin, E. F., Fricke, W. F., Gajer, P., Crabtree, J., Sebaihia, M., Thomson, N. R. \& other authors (2008). The pangenome structure of Escherichia coli: comparative genomic analysis of $E$. coli commensal and pathogenic isolates. J Bacteriol 190, 6881-6893.

Schlager, T. A., Whittam, T. S., Hendley, J. O., Wilson, R. A., Bhang, J., Grady, R. \& Stapleton, A. (2000). Expression of virulence factors among Escherichia coli isolated from the periurethra and urine of children with neurogenic bladder on intermittent catheterization. Pediatr Infect Dis J 19, 37-41.

Schlager, T. A., Johnson, J. R., Ouellette, L. M. \& Whittam, T. S. (2008). Escherichia coli colonizing the neurogenic bladder are similar to widespread clones causing disease in patients with normal bladder function. Spinal Cord 46, 633-638.

Shaaban, A. A., el-Nono, I. H., Abdel-Rahman, M. \& Ghoneim, M. A. (1992a). The urodynamic characteristics of different ileal reservoirs: an experimental study in dogs. J Urol 147, 197-200.

Shaaban, A. A., Gaballah, M. A., el-Diasty, T. A. \& Ghoneim, M. A. (1992b). Urethral controlled bladder substitution: a comparison between the intussuscepted nipple valve and the technique of Le Duc as antireflux procedures. J Urol 148, 1156-1161.

Skyberg, J. A., Johnson, T. J., Johnson, J. R., Clabots, C., Logue, C. M. \& Nolan, L. K. (2006). Acquisition of avian pathogenic Escherichia coli 
plasmids by a commensal E. coli isolate enhances its abilities to kill chicken embryos, grow in human urine, and colonize the murine kidney. Infect Immun 74, 6287-6292.

Studer, U. E., Casanova, G. A. \& Zingg, E. J. (1991). Continent urinary diversion by an ileal bladder substitute. Prog Clin Biol Res 370, 59-66.

Studer, U. E., Danuser, H., Hochreiter, W., Springer, J. P., Turner, W. H. \& Zingg, E. J. (1996). Summary of 10 years' experience with an ileal low-pressure bladder substitute combined with an afferent tubular isoperistaltic segment. World J Urol 14, 29-39.

Suriano, F., Gallucci, M., Flammia, G. P., Musco, S., Alcini, A., Imbalzano, G. \& Dicuonzo, G. (2008). Bacteriuria in patients with an orthotopic ileal neobladder: urinary tract infection or asymptomatic bacteriuria? BJU Int 101, 1576-1579.

Tettelin, H., Riley, D., Cattuto, C. \& Medini, D. (2008). Comparative genomics: the bacterial pan-genome. Curr Opin Microbiol 11, 472-477.

Touchon, M., Hoede, C., Tenaillon, O., Barbe, V., Baeriswyl, S., Bidet, P., Bingen, E., Bonacorsi, S., Bouchier, C. \& other authors (2009). Organised genome dynamics in the Escherichia coli species results in highly diverse adaptive paths. PLoS Genet 5, e1000344.

Valentiner-Branth, P., Steinsland, H., Fischer, T. K., Perch, M., Scheutz, F., Dias, F., Aaby, P., Mølbak, K. \& Sommerfelt, H. (2003). Cohort study of Guinean children: incidence, pathogenicity, conferred protection, and attributable risk for enteropathogens during the first 2 years of life. J Clin Microbiol 41, 4238-4245.
Wei, J., Goldberg, M. B., Burland, V., Venkatesan, M. M., Deng, W., Fournier, G., Mayhew, G. F., Plunkett, G., III, Rose, D. J. \& other authors (2003). Complete genome sequence and comparative genomics of Shigella flexneri serotype 2a strain 2457T. Infect Immun 71, 2775-2786.

Welch, R. A., Burland, V., Plunkett, G., III, Redford, P., Roesch, P., Rasko, D., Buckles, E. L., Liou, S. R., Boutin, A. \& other authors (2002). Extensive mosaic structure revealed by the complete genome sequence of uropathogenic Escherichia coli. Proc Natl Acad Sci U S A 99, 17020-17024.

Whittam, T. S., Ochman, H. \& Selander, R. K. (1983). Geographic components of linkage disequilibrium in natural populations of Escherichia coli. Mol Biol Evol 1, 67-83.

Whittam, T. S., Wolfe, M. L., Wachsmuth, I. K., Orskov, F., Orskov, I. \& Wilson, R. A. (1993). Clonal relationships among Escherichia coli strains that cause hemorrhagic colitis and infantile diarrhea. Infect Immun 61, 1619-1629.

Wullt, B., Agace, W. \& Mansson, W. (2004). Bladder, bowel and bugs bacteriuria in patients with intestinal urinary diversion. World J Urol 22, 186-195.

Zerbino, D. R. \& Birney, E. (2008). Velvet: algorithms for de novo short read assembly using de Bruijn graphs. Genome Res 18, 821-829.

Edited by: S. D. Bentley 